\title{
A Comparative Gene Map of the Horse (Equus caballus)
}

\author{
Alexandre R. Caetano, ${ }^{1,2,6}$ Yow-Ling Shiue, ${ }^{1,2,6}$ Leslie A. Lyons, ${ }^{3}$ Steven J. O'Brien, ${ }^{3}$ \\ Thomas F. Laughlin, ${ }^{4}$ Ann T. Bowling, ${ }^{1,5}$ and James D. Murray ${ }^{2,5,7}$ \\ ${ }^{1}$ Veterinary Genetics Laboratory, University of California Davis, Davis, California 95616-8744 USA; ${ }^{2}$ Department of Animal \\ Science, University of California Davis, Davis, California 95616 USA; ${ }^{3}$ Laboratory of Genomic Diversity, National Cancer \\ Institute, Frederick Cancer Research and Development Center, Frederick, Maryland 21702-1201 USA; ${ }^{4}$ Department of \\ Biological Sciences, East Tennessee State University, Johnson City, Tennesee 37614 USA; ${ }^{5}$ Department of Population Health \\ and Reproduction, University of California Davis, Davis, California 95616 USA
}

\begin{abstract}
A comparative gene map of the horse genome composed of 127 loci was assembled based on the new assignment of 68 equine type I loci and on data published previously. PCR primers based on consensus gene sequences conserved across mammalian species were used to amplify markers for assigning 68 equine type I loci to 27 horse synteny groups established previously with a horse-mouse somatic cell hybrid panel (SCHP, UC Davis). This increased the number of coding genes mapped to the horse genome by over 2-fold and allowed refinements of the comparative mapping data available for this species. In conjunction with 57 previous assignments of type I loci to the horse genome map, these data have allowed us to confirm the assignment of 24 equine synteny groups to their respective chromosomes, to provisionally assign nine synteny groups to chromosomes, and to further refine the genetic composition established with Zoo-FISH of two horse chromosomes. The equine type I markers developed in this study provide an important resource for the future development of the horse linkage and physical genome maps.
\end{abstract}

The rapid progress being made in the development of genetic maps for humans and mice (Hudson et al. 1995; Stewart et al. 1997; Rhodes et al. 1998) has led to a recent boom in the construction of genome maps for a number of domesticated mammalian species of economic importance (Bishop et al. 1994; Archibald et al. 1995; O'Brien et al. 1997a; de Gortari et al. 1998). Many recent technological advances have contributed to the generation of these data which will greatly increase our ability to find and isolate genes that lead to genetic diseases and/or have an effect on economically important production traits of livestock (Cockett et al. 1994; Georges and Andersson 1996; Grobet et al. 1997).

Molecular markers based on repetitive or anonymous DNA sequences (Type II markers, O'Brien et al. 1993) such as microsatellites, Random Amplified Polymorphic DNA (RAPDs), and Amplified Fragment Length Polymorphisms (AFLPs) have been used extensively to saturate the genetic maps of various species because of their technical advantages and high degree of polymorphism (O'Brien et al. 1993; Georges and Andersson 1996; Andersson et al. 1996). Type II markers, however, are seldom informative across mammalian orders and therefore do not allow for the comparison of genetic maps from different species $\left(\mathrm{O}^{\prime}\right.$ Brien et al. 1993; Georges and Andersson 1996; Andersson et al.

\footnotetext{
${ }^{6}$ These authors contributed equally to this work. ${ }^{7}$ Corresponding author.
}

E-MAIL jdmurray@ucdavis.edu; FAX (530) 752-3179.
1996). Conversely, molecular markers for functional genes conserved across species (Type I anchor loci, O'Brien et al. 1993) can be used for this purpose.

In conjunction with chromosome painting (ZooFISH) studies, mapping studies with type I loci have revealed that genomes from related species have a high degree of synteny conservation (O'Brien et al. 1993; Andersson et al. 1996; Wakefield and Graves 1996). This information forms the basis for comparative genome mapping, a discipline that allows the prospect of using information from highly characterized genomes to study genetic phenomena in map-poor species (O'Brien et al. 1993; Georges and Andersson 1996; Andersson et al. 1996; Wakefield and Graves 1996).

Although the horse genome is not as highly characterized as the genomes of other domestic animals, much progress has been made recently. Somatic cell hybrid (SCH) panels have been used to make synteny assignments of 240 type II markers (Bailey et al. 1995; Shiue et al. 1999), many of which were physically assigned to horse chromosomes by FISH (Sakagami et al. 1995; Tozaki et al. 1995; Breen et al. 1997; Godard et al. 1997, 1998). In addition, the first low-resolution microsatellite-based linkage maps of the horse have been published (Lindgren et al. 1998; Guérin et al. 1999). In the horse, a framework for comparative mapping has been established with chromosome painting studies (Raudsepp et al. 1996, 1997; Rettenberger et al. 1996), FISH assignments of type I loci (Lear et al. 1998a,b,c; 
Godard et al. 1998), and by synteny assignments of type I loci with SCH panels (Williams et al. 1993; Bailey et al. 1995; Caetano et al. 1999a,b). Using human chromosome-specific probes, Zoo-FISH was used to determine that a minimum of 21 chromosomal breaks, followed by the appropriate rearrangements, are necessary to reconstruct the horse karyotype relative to the human karyotype (Raudsepp et al. 1996; Rettenberger et al. 1996; Raudsepp et al. 1997; Chaudhary et al. 1998). However, in addition to these advancements in the equine genome map, a larger group of type I anchor loci needs to be mapped to allow for thorough comparisons with maps from model species and the study of the fine intrachromosomal gene structure of the horse genome.

Despite their value for comparative mapping, the incorporation of type I anchor loci to genome maps of multiple species has not been used extensively because of technical reasons. Large collections of PCR primer sets for type I loci [i.e. universal mammalian-sequencetagged sites (UM-STS), comparative anchor tagged sequences (CATS) have been designed to amplify mammalian sequences by taking advantage of public sequence databases (Venta et al. 1996; Lyons et al. 1997). In this report we present the synteny assignment of 68 equine type I loci by analysis of a horse-mouse $\mathrm{SCH}$ panel with PCR-based markers generated with universal primers for mammalian genes.

\section{RESULTS}

\section{Development of PCR-Based Markers for Equine Type I loci}

In addition to amplifying gene-specific fragments, a key feature necessary for the effective use of PCR-based markers in synteny mapping with interspecific $\mathrm{SCH}$ panels is the ability to differentiate between fragments amplified from the donor species (i.e. horse) and the murine background in each hybrid cell line. Most of the primers used in this study were designed to anneal to conserved short exon sequences flanking introns, which should therefore result in the amplification of fragments of different sizes and/or sequences in the two species.

A total of 289 previously published primers for mammalian type I loci (Venta et al. 1996; Lyons et al. 1997) were tested for their ability to amplify horsespecific fragments useful for synteny mapping with a SCH panel. PCR conditions were optimized to amplify markers for 42 equine type I loci (Table 1). Digestions of PCR products from mouse and horse using restriction enzymes were used to obtain gene-specific equine markers with an additional 26 primer sets. In one instance (RB1), an equine-specific primer was designed (Table 1) based on the sequence obtained from a fragment amplified from horse with primers published pre- viously for this gene (Venta et al. 1996). The remaining primers amplified no fragments (5 primers), multiple equine fragments (180 primers), or fragments from horse and mouse that could not be differentiated with the techniques used (36 primers).

\section{Sequencing PCR Products}

To verify that the PCR products used for making synteny assignments correspond to the expected equine gene homologs, a total of 50 isolated PCR fragments amplified from a thoroughbred horse were cloned and sequenced. The remaining 18 horse PCR products were gel-isolated and partially sequenced by direct sequencing (Table1). All of the nucleotide sequences obtained were used to search GenBank with the BLAST routine and confirm the identity of 66 of the genes mapped. In two instances (KRAS2, SOD2), the cloned equine PCR fragment lacked the expected intron sequence, sug gesting that the PCR products mapped are pseudogenes.

Many of the primers that amplified horse-specific fragments were designed to target specific genes from large paralogous gene families. Several of the isolated PCR products cloned were found to contain multiple fragments of highly homologous sequences, most likely amplified from different syntenic isoforms of paralogous genes (Table 1). For example, the MYH6 primers (Lyons et al. 1997) amplified two equine fragments that are 97\% identical at the expected exon regions. These equine fragments are highly homologous to the human myosin heavy polypeptide alpha and beta genes (MYH6 and MYH7, respectively), which are closely linked and show 93\% sequence identity. Similarly, the primers used to amplify two highly homologous equine fragments from the equine $I L 1 B$ gene (92\% identity) were originally designed to amplify IL1A (Lyons et al. 1997), two genes that are closely linked in human Chromosome 2. Therefore, although these markers were useful for determining the equine synteny assignment of the respective loci, further characterization will be necessary for determining which gene sequence was amplified from these paralogous gene families in the horse.

In one instance (SST), the equine PCR product contained fragments with the expected sequence in addition to fragments with completely unrelated sequence, except for the primer binding sites. Primers specific to the anonymous equine fragment were designed (F-5'TTTCCATGGACTTATTTCCC-3'/R-5' -TCCCTTGTTACCTGGAGTATG-3'). The PCR product amplified with these primers was only found in the $\mathrm{SCH}$ clonal lines where the SST product was found, suggesting this unrelated sequence is syntenic with the equine SST gene.

\section{SCH Panel Analysis with Equine PCR-based} Type I Loci

The genome of the horse consists of 31 autosomes plus 
Table 1. PCR Conditions for Amplifying 68 Equine Type I Loci with Universal Primers

\begin{tabular}{|c|c|c|c|c|c|c|c|c|}
\hline Hsa & UCD & Symbol & Locus name & $\begin{array}{c}\text { Anl } \\
\text { T. }{ }^{\circ} \mathrm{C}\end{array}$ & $\begin{array}{l}\mathrm{MgCl} \\
(\mathrm{mM})\end{array}$ & R.E. & $\begin{array}{l}\text { Size } \\
\text { (bp) }\end{array}$ & $\begin{array}{c}\text { Accession } \\
\text { no. }^{a}\end{array}$ \\
\hline $1 \mathrm{p} 36.2$ & 2 & NPPA & natriuretic peptide precursor A & 50 & 2 & Hinfl & 305 & AF134229 \\
\hline $1 \mathrm{p} 13.1$ & 5 & NGFB & nerve growth factor, $\beta$ & 53 & 2.5 & Rsal & 158 & AF134228 \\
\hline $1 q 21$ & 5 & $G B A$ & glucosidase, $\beta$ acid & 53 & 2 & Rsal & 345 & AF134220 \\
\hline $1 q 23-q 25.1$ & 5 & AT3 & antithrombin III & 55 & 2 & - & 187 & AF134055 \\
\hline $1 \mathrm{q} 31$ & 5 & LAMC1 & laminin, $C-1$ & 50 & 2 & - & $\sim 1400$ & AF134225, 6 \\
\hline $2 \mathrm{p} 21$ & 15 & SPTBN $1^{\mathrm{b}}$ & spectrin, $\beta$, nonerythrocytic 1 & 50 & 2 & - & 975 & AF130782 \\
\hline $2 q 12-q 14$ & 15 & PAX8 & paired box homeotic gene 8 & 55 & 2 & HaellI & 147 & AF130778 \\
\hline $2 q 14$ & 15 & $I L 1 b^{c, d}$ & interleukin-1 $\beta$ & 55 & 1 & HindIII & $\sim 906$ & AF130767, 8 \\
\hline $2 q 24-q 32$ & 18 & CHRNA & cholinergic receptor, nicotinic, $\alpha$ & 58 & 1 & - & 405 & AF130750 \\
\hline $2 q 31-q 32$ & 18 & NEB & nebulin & 56 & 1 & Pvull & $\sim 2000$ & AF130774, 5 \\
\hline $2 q 33-q 34$ & $\mathrm{D}$ & CHRNG & cholinergic receptor, nicotinic, $\gamma$ & 60 & 1 & - & 264 & AF130751 \\
\hline $2 q 34$ & D & FN1 & fibronectin 1 & 55 & 2 & - & 1009 & AF130764 \\
\hline $3 p 24.3-p 24.2$ & 16 & $R A R B$ & retonic acid receptor, $\beta$ & 55 & 3 & Cfol & 208 & AF13424 \\
\hline $3 p 21.33$ & 16 & $G L B 1^{\mathrm{b}}$ & galactosidase, $\beta-1$ & 50 & 2 & Rsal & 429 & AF130765 \\
\hline $3 q 21-q 24$ & 16 & $C P$ & ceruloplasmin & 55 & 2.5 & - & $\sim 1500$ & AF134057 \\
\hline $3 q 21-q 24$ & 16 & $\mathrm{RHO}$ & rhodopsin & 55 & 2 & Rsal & 327 & AF130780 \\
\hline $3 q 28$ & 19 & $S S T^{\mathrm{e}}$ & somatostatin & 50 & 1 & - & 931 & AF130783 \\
\hline $4 q 22$ & 3 & $A D H 2$ & alcohol dehydrogenase 2 & 52 & 1 & - & 295 & AF133272 \\
\hline $4 q 22$ & 3 & $A D H 3$ & alcohol dehydrogenase 3 & 52 & 2 & - & $>2000$ & AF134056 \\
\hline $4 q 28$ & 2 & FGG & fibrinogen, $\gamma$ polypeptide & 53 & 2 & - & $\sim 1100$ & AF134218, 9 \\
\hline $5 q^{32}-q^{34}$ & 14 & $A D R B 2$ & adrenergic receptor, $\beta-2$ & 55 & 2 & Hinfl & 354 & AF130746 \\
\hline $6 p 24-p 23$ & 20 & $E D N I^{\mathrm{b}}$ & endothelin-1 & 58 & 2 & - & 266 & AF130760 \\
\hline $6 q 13$ & 20 & $\operatorname{COL9a1^{b}}$ & collagen, type IX, $\alpha-1$ & 50 & $\overline{2}$ & - & 292 & AF130755 \\
\hline $6 q 21.1-q 23$ & 10 & CGA & chorionic gonadotropin, $\alpha$ chain & 45 & 4 & - & 242 & AF130749 \\
\hline $6 q 21-q 22.3$ & 10 & $\mathrm{COL} 10^{\mathrm{b}}$ & collagen, type $X, \alpha 1$ & 50 & 2 & PVull & 365 & AF130754 \\
\hline $6 \mathrm{q} 25.3$ & 14 & SOD $2 p s^{g}$ & superoxide dismutase 2 & 55 & 2 & - & 154 & AF130781 \\
\hline 7p15-p14 & 4 & TCRG & t cell antigen receptor, $\gamma$ subunit & 42 & 2.5 & Hinfl & 252 & AF134235 \\
\hline $7 q 11.2$ & 13 & $E L N^{\mathrm{b}}$ & elastin & 58 & 1 & - & 263 & AF130761 \\
\hline $7 q 21.11$ & 13 & GUSB & glucuronidase $\beta$ & 53 & 2 & Sau3Al & 382 & AF134223 \\
\hline $7 q 36$ & 4 & EN2 & Engrailed 2 & 53 & 2 & Pvull & 189 & AF134060 \\
\hline $8 q 24.2-q 24.3$ & 9 & TG & thyroglobulin & 58 & 1 & - & 749 & AF130785 \\
\hline $9 p 22$ & 23 & IFNA1 & interferon, $\alpha-1$ & 53 & 2 & Pvull & 373 & AF135017 \\
\hline $9 p 21$ & 23 & IFNB1 & interferon, $\beta-1$ & 55 & 2 & Haelll & 450 & AF134227 \\
\hline $9 p 13$ & 23 & CNTFR & ciliary neurotrophic f. recept. & 57 & 2.5 & - & 456 & AF134058 \\
\hline $9 q 31$ & 25 & $T X N$ & thioredoxin & 55 & 2 & Hinfl & $\sim 780$ & AF134237 \\
\hline $9 q 34$ & 25 & GRP78 & glucose-regulated protein & 55 & 2 & Pvull & $\sim 740$ & AF134222 \\
\hline $10 p 13$ & 29 & VIM & vimentin & 57 & 2 & - & $\sim 1230$ & AF135018 \\
\hline $11 p 15.5$ & 7 & $H B B$ & hemoglobin, $\beta$ & 50 & 2.5 & - & 171 & AF134224 \\
\hline 11 p15.3-p15.1 & 7 & PTH & parathyroid hormone & 55 & 2 & - & 311 & AF134233 \\
\hline 11 cen-q13 & 12 & $A D R B K 1$ & adrenergic receptor $\beta$ kinase I & 53 & 1 & - & 328 & AF134059 \\
\hline $11 q^{13}$ & 12 & $C D 20^{\mathrm{b}}$ & CD20 antigen & 55 & 2 & - & 1007 & AF130748 \\
\hline $11 q 23$ & 7 & DRD2 & dopamine receptor D2 & 50 & 2.5 & - & $\sim 1300$ & AF134061, 2 \\
\hline $11 q 23.3$ & 7 & THY1 & Thy-1 T-cell surface antigen & 50 & 2 & - & $\sim 800$ & AF134236 \\
\hline
\end{tabular}

(Continued on following page.)

the $\mathrm{X}$ and $\mathrm{Y}$ sex-chromosomes. Previous work with the UC Davis horse-mouse SCH panel established 33 equine synteny groups. A total of 25 synteny groups were assigned to their respective horse chromosomes by correlation with microsatellites and type I loci mapped previously by FISH (Shiue et al. 1999; Caetano et al. 1999a), with microsatellites assigned by DNA typing of trisomic horses (Bowling et al. 1997) and with sex-chromosome type I markers (Shiue 1999). Chromosome assignments of synteny groups were made based on a range of one to six physically assigned loci.

The 68 markers for equine type I loci we charac- 
Table 1. (Continued)

\begin{tabular}{|c|c|c|c|c|c|c|c|c|}
\hline Hsa & UCD & Symbol & Locus name & $\begin{array}{l}\text { Anl } \\
\text { T. }{ }^{\circ} \mathrm{C}\end{array}$ & $\begin{array}{l}\mathrm{MgCl} \\
(\mathrm{mM})\end{array}$ & R.E. & $\begin{array}{l}\text { Size } \\
\text { (bp) }\end{array}$ & $\begin{array}{c}\text { Accession } \\
\text { no. }^{a}\end{array}$ \\
\hline $12 \mathrm{p} 12.1$ & 9 & $K R A S 2 p s^{g}$ & Kirsten murine sarc. 2 viral onc., ps. & 58 & 1 & - & 329 & AF130769 \\
\hline $12 q 13.3$ & D & PFKM & phosphofructokinase $\mathrm{m}$ & 60 & 2 & - & $\sim 1600$ & AF134230, 1 \\
\hline $12 q 14$ & D & IFNG & Interferon $\gamma$ & 45 & 2 & - & 325 & AF130766 \\
\hline $12 q 24.2$ & 6 & TCF1 & transcription factor 1 & 58 & 1 & - & 602 & AF130784 \\
\hline $13 q 14.1 q-14.2$ & 17 & $R B 1^{\mathrm{f}}$ & retinoblastoma & 55 & 2 & - & 705 & AF130779 \\
\hline $14 q 11.2$ & 1 & $\mathrm{CH} Y^{\mathrm{b}}$ & chymase- mast cell & 58 & 2 & - & 697 & AF130752 \\
\hline $14 q 12$ & 1 & $M Y H 6^{d}$ & myosin, heavy pol. $6 \& /$ or 7 , see text & 58 & 2 & Rsal & $\sim 650$ & AF130771, 2 \\
\hline $15 q 22-q$ ter & 1 & CYP1A2 & cytochrome P450, subf. I, polyp. 2 & 50 & 2.5 & Hinfl & 278 & AF134063 \\
\hline $15 q 26.1$ & 1 & $F E S^{b}$ & feline sarcoma virus homologue & 58 & 2 & - & 510 & AF130763 \\
\hline $17 q 11.2$ & 11 & EVI2A & ecotropic viral int. site $2 \mathrm{~A}$ & 55 & 4 & - & 156 & AF130762 \\
\hline $17 q 11.2$ & 11 & NF1 & neurofibromatosis I & 58 & 2 & - & 380 & AF130776 \\
\hline $17 q 25$ & 11 & P4HB & prolyl-4 hydroxylase $\beta$ & 62 & 2 & - & 460 & AF130777 \\
\hline 17 & 11 & MYL4 & myosin light chain 4 & 58 & 2 & - & 682 & AF130773 \\
\hline $18 p 11.32$ & 6 & $T S^{b}$ & thymidylate synthetase & 55 & 1 & - & 539 & AF130786 \\
\hline $19 q 13$ & 10 & $C K M^{\mathrm{b}}$ & creatine kinase, muscle & 58 & 1 & - & 819 & AF130753 \\
\hline 20pter-p12 & 22 & PRNP & prion protein & 55 & 1.5 & - & 191 & AF134232 \\
\hline $20 q 13.2$ & 22 & GNAS1 & guanine nuc. binding protein & 50 & 1.5 & Rsal & 324 & AF134221 \\
\hline $20 q 13.11$ & 22 & $A D A$ & adenosine deaminase & 50 & 1.5 & - & $\sim 1350$ & AF135790 \\
\hline $21 q 22.3$ & 26 & ETS2 & avian erythroblastosis virus onc. 2 & 55 & 2 & Hinfl & $\sim 1000$ & AF134064 \\
\hline $22 q 13.1$ & C & $C Y P 2 D^{d}$ & cytochrome P450, subfamily IID & 58 & 2 & - & 980 & AF130756- 9 \\
\hline $22 q 13.31-$ qter & C & $A R S A$ & arylsulfatase $A$ & 58 & 2 & - & 247 & AF130747 \\
\hline Xp11.21 & $\mathrm{x}$ & ALAS2 & $\Delta$-aminolevulinate synthase & 53 & 2 & HaellI & $\sim 800$ & AF133200 \\
\hline Xq27.1-q27.2 & $\mathrm{x}$ & F9 & coagulation factor IX & 55 & 2 & - & 453 & AF133201 \\
\hline $\mathrm{Xq} 28$ & $\mathrm{x}$ & $B G N$ & biglycan & 57 & 2 & Hinfl & $\sim 710$ & AF135019, 0 \\
\hline $\mathrm{Xq} 28$ & $\mathrm{X}$ & G6PD & glucose-6-phosphate dehydro. & 55 & 2 & Hinfl & $\sim 750$ & AF133202 \\
\hline
\end{tabular}

All primers used in this study were published by Lyons and coworkers (1997), with the following exceptions:

a Multiple accession numbers indicated sequences obtained from the $5^{\prime}$ and $3^{\prime}$ ends of partially sequenced PCR products, or multiple highly homologous sequences most likely amplified from different syntenic isoforms of paralogous genes (see text).

bPrimers published by Venta and associates (1996).

'The primers used to amplify equine IL1B were originally designed to amplify IL $1 A$ (see text).

dThese primers amplify multiple equine fragments of different but highly homologous sequences (see text).

eAmplifies a fragment of unknown sequence in addition to the equine SST gene (see text).

'The forward primer used to amplify equine RB1 ( 5 '=TTTGATATCGAAGGGTCTGAC-3') was designed in this study and the reverse primer was as in Venta et al. (1996).

${ }^{9}$ These primers amplify equine sequences lacking expected introns and therefore are thought to be horse pseudogenes from the respective loci.

terized were mapped to 27 different synteny groups (Table 2). Forty-five of these markers were mapped to 18 synteny groups that had been assigned previously to specific horse chromosomes. The remaining 23 markers were mapped to 9 synteny groups which are not presently assigned to equine chromosomes.

\section{DISCUSSION}

Sixty-eight makers for equine type I loci were characterized and mapped by SCH panel analysis. These data, combined with 26 type I loci previously assigned with the UC Davis SCH panel (Caetano et al. 1999a,b) and
32 type I loci mapped by other groups working on the characterization of the horse gene map, provide the most comprehensive comparative mapping data currently available for the horse genome (Table 3), when considered in conjunction with chromosome painting studies of the horse karyotype (Raudsepp et al 1996, 1997; Rettenberger et al. 1996; Chaudhary et al. 1998). These data have allowed us to confirm the assignment of 24 equine synteny groups to their respective chromosomes, to provisionally assign nine synteny groups, and to further refine the genetic composition of two horse chromosomes established previously with ZooFISH.

\section{Genome Research}


Table 2. Correlation Values of 68 Equine Type I Loci with Markers Previously Assigned to UCD Davis Syntenic Groups and/or Horse Chromosomes

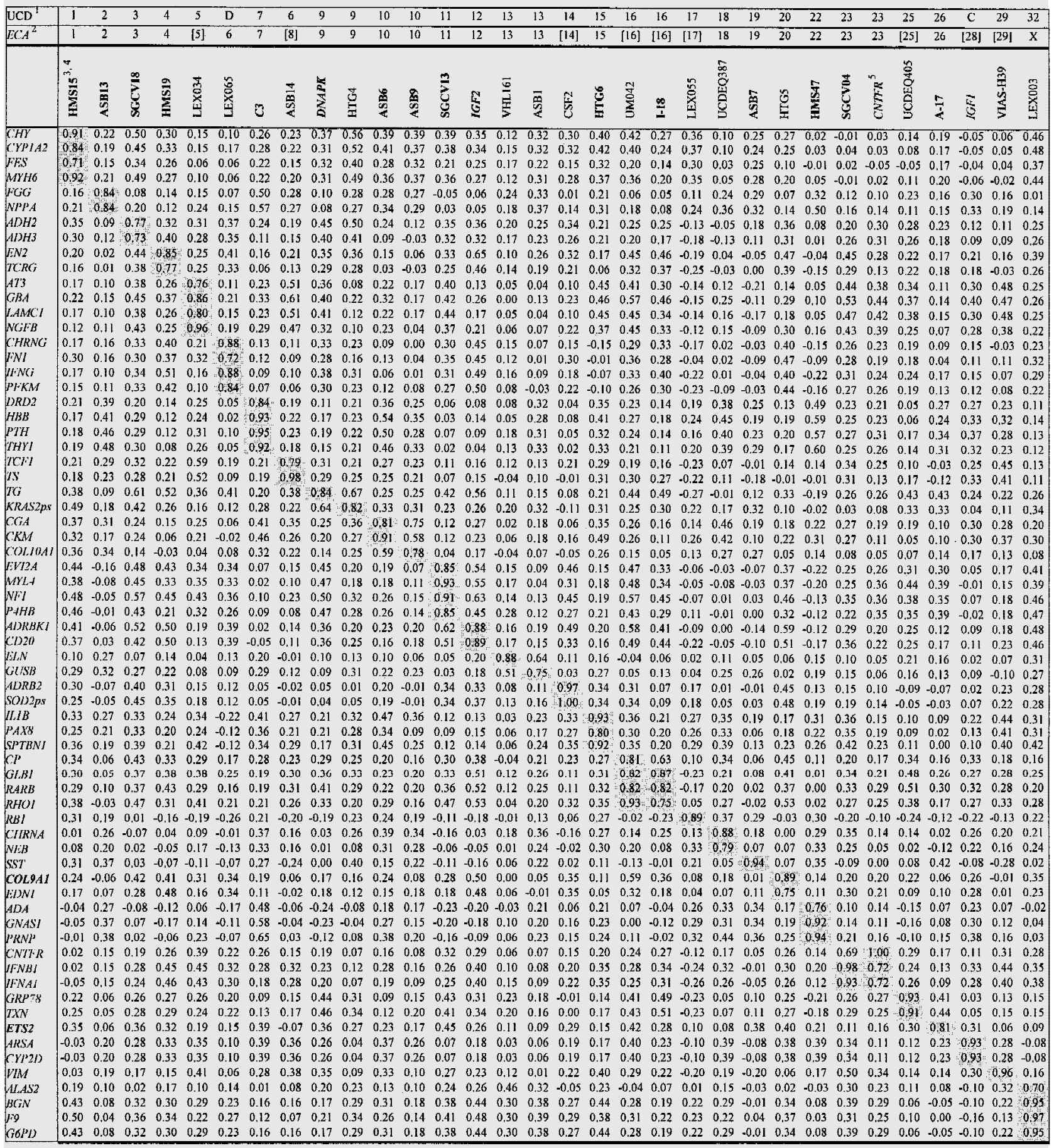

Significant correlation values are indicated in the shaded areas.

${ }^{1}$ Indicates the UCD syntenic group number.

${ }^{2} \mathrm{~A}$ number in this row indicates the syntenic group has been assigned to the corresponding horse chromosome. Numbers in square brackets indicate the syntenic group is provisionally assigned to the corresponding chromosome (see text).

${ }^{3}$ Markers in this row were chosen as reference loci for the respective synteny groups (see Shiue et al. 1999; Caetano et al. 1999a,b). ${ }^{4}$ Markers in bold have been assigned to the respective horse chromosomes by FISH.

${ }^{5}$ CNTFR is not significantly correlated with any of the microsatellite markers mapped to UCD23, therefore this column was added to show the significant correlation to two equine genes added to this synteny group in this study (IFNB1, IFNA1). 
Table 3. Human-Horse Comparative Gene Map Using Synteny, Linkage and in Situ Hybridization (127 Genes)

\begin{tabular}{|c|c|c|c|c|c|c|c|c|}
\hline $\mathrm{Hsa}^{\mathrm{a}}$ & Locus & Locus name & $E C A^{\mathbf{b}}$ & Method $^{c}$ & $B t a^{\mathrm{d}}$ & $M m u^{\mathrm{e}}$ & $F_{c a} a^{f}$ & Ssc $^{\mathrm{g}}$ \\
\hline $1 p 36.2$ & NPPA & natriuretic peptide precursor, A & 2 & $S$ & 16 & 4 & - & - \\
\hline 1 p36.2-p36.13 & $P G D$ & phosphogluconate dehydrogenase & $2 p 12$ & $\mathrm{I}, \mathrm{L}$ & 16 & 4 & C1 & $6 q 2.2 q 2.5$ \\
\hline $1 \mathrm{p} 31$ & PGM1 & phosphoglucomutase 1 & 5 & $\mathrm{~L}$ & 3 & 5 & C1 & 6 \\
\hline $1 \mathrm{p} 13.1$ & $N G F B$ & nerve growth factor, $\beta$ & 5 & $S$ & 3 & 3 & - & $4 q 1.6-q 2.3$ \\
\hline $1 q 21$ & $G B A$ & glucosidase, $\beta$, acid & 5 & $S$ & 3 & 3 & - & 4 \\
\hline $1 q 23-q 25.1$ & AT3 & antithrombin III & 5 & S & 16 & 1 & - & - \\
\hline $1 \mathrm{q} 31$ & LAMC1 & laminin, C1 & 5 & S & 16 & 1 & - & - \\
\hline $2 \mathrm{p} 25$ & ODC1 & ornithine decarboxylase & $15 q 27$ & I & - & 12 & - & - \\
\hline $2 \mathrm{p} 21$ & $C A D$ & carbamoylphosphate synthetase & $15 q 25$ & 1 & - & 1 & - & - \\
\hline $2 \mathrm{p} 21$ & SPTBN1 & spectrin, $\beta$, nonerythrocytic 1 & 15 & S & - & 11 & - & - \\
\hline $2 q^{14}$ & $I L 1 B$ & interleukin $1, \beta$ & 15 & S & 2 & 2 & & $3 q 1.1-q 1.4$ \\
\hline $2 q^{12}-q^{14}$ & PAX8 & human paired domain & 15 & $S$ & - & 2 & - & - \\
\hline $2 q 21$ & $L C T$ & lactase-phlorizin hydrolase & $15 q 21$ & l & - & 1 & - & $15 q 1.3$ \\
\hline $2 q 32.1$ & MSTN & myostatin & 18 & S & 2 & 1 & - & - \\
\hline $2 q 24-q 32$ & CHRNA & cholinergic receptor, nicotin, $\alpha$ & 18 & $S$ & 2 & 2 & - & - \\
\hline $2 q 31-q 32$ & NEB & nebulin & 18 & S & 2 & 2 & - & - \\
\hline $2 q 33-34$ & CHRNG & cholinergic receptor, nicotin, $\gamma$ & [6] & $S$ & 2 & 1 & - & - \\
\hline $2 q 34$ & FN1 & fibronectin 1 & [6] & S & $\overline{2}$ & 1 & - & - \\
\hline $3 p 24.3-24.2$ & $R A R B$ & retinoic acid receptor, $\beta$ & 16 & $S$ & - & 14 & - & - \\
\hline $3 p 21.33$ & GLB1 & galactosidase, $\beta, 1$ & 16 & $S$ & - & 9 & B3 & - \\
\hline $3 q 21-q 24$ & $\mathrm{RHO}$ & rhodopsin & 16 & $S$ & 22 & 6 & - & - \\
\hline $3 q 21$ & $T F$ & transferrin & $16 q 23$ & $S$ & 1 & 9 & - & $13 q 3.1$ \\
\hline $3 q 21-q 23$ & $L T F$ & lactotransferrin & $16 q$ & I & 22 & 9 & - & - \\
\hline $3 q 21-q 24$ & $C P$ & ceruloplasmin & 16 & $S$ & 1 & 9 & - & $13 q 3.2-q 3.3$ \\
\hline $3 q 28$ & SST & somatostatin & 19 & S & 1 & 16 & - & - \\
\hline $4 p 16.3$ & FGFR3 & fibroblast growth factor receptor & 3 & S & 6 & 5 & - & - \\
\hline $4 q 11-q 13$ & $A L B$ & albumin & $3 q 14$ & $S, I$ & 6 & 5 & - & $8 q 1.2$ \\
\hline $4 q 12$ & $G C$ & group specific component & 3 & $\mathrm{~L}$ & 6 & 5 & - & - \\
\hline $4 q 12$ & KIT & mast cell growth factor receptor & $3 q 21$ & $\mathrm{~L}, \mathrm{I}$ & 6 & 5 & B1 & 8p12-p21 \\
\hline $4 q 12$ & PDGFRA & platelet derived growth factor $\mathrm{A}$ & $3 q 21$ & $\mathrm{I}, \mathrm{L}$ & 6 & 5 & - & $8 p 12$ \\
\hline $4 q 22$ & $A D H 2$ & alcohol dehydrogenase 2 & 3 & $S$ & 6 & 3 & - & - \\
\hline $4 q 22$ & $A D H 3$ & alcohol dehydrogenase 3 & 3 & $S$ & 6 & 3 & - & 8 \\
\hline $4 q 26-q 27$ & IL2 & interleukin 2 & 2 & $S$ & 17 & 3 & B1 & 8 \\
\hline $4 q 28$ & FGG & fibrinogen, $\gamma$ polyp & 2 & S & 17 & 3 & - & 8 \\
\hline $5 p 13$ & $I L 7 R$ & interleukin receptor 7 & 21 & $S$ & - & 15 & - & - \\
\hline 5p13-p12 & $G H R$ & growth hormone receptor & 21 & S & 20 & 15 & - & $16 q 1.3-q 1.4$ \\
\hline $5 p 14$ & C9 & complement component 9 & 21 & S & 1 & - & - & $16 q 14$ \\
\hline $5 q 11-q 12$ & CTLA3 & cytotoxic T-1 assoc serine es 3 & 21 & $\mathrm{~S}$ & - & 13 & - & - \\
\hline $5 q 13$ & $H E X B$ & hexoseaminidase $b$ & 14 & S & 20 & 13 & - & - \\
\hline $5 q 21-q 23$ & CAMK4 & calmodulin depend. Prot. kinase 4 & 14 & $S$ & - & 18 & - & - \\
\hline $5 q 31.1$ & CSF2 & colony stimulating factor 2 & 14 & $S$ & 7 & 11 & - & 5 \\
\hline $5 q 31-q 32$ & SPARC & secreted pro, ac, cyst-rich & 14 & $S$ & 7 & 11 & - & - \\
\hline $5 q 32-34$ & $A D R B 2$ & adrenergic receptor, $\beta 2$ & 14 & S & 7 & 18 & - & - \\
\hline $5 q 33.2-q 33.3$ & CSF1R & colony stimulating factor 1 receptor & 14 & S & 7 & 18 & A1 & - \\
\hline 6pter-p21.1 & CLPS & colipase & 20 & $S$ & - & 17 & - & 7 \\
\hline $6 \mathrm{p} 25-\mathrm{p} 24$ & F13 & coagulation factor $13, \mathrm{~A}$ & $20 q 13$ & $\mathrm{~L}, \mathrm{I}$ & 23 & - & - & 4 \\
\hline $6 \mathrm{p} 21.2$ & PIM1 & Pim-1 oncogene & $20 q 24$ & 1 & - & 17 & B2 & - \\
\hline $6 \mathrm{p} 21$ & $H L A$ & major histocompatibility complex & $20 q$ & $\mathrm{~L}, \mathrm{I}, \mathrm{S}$ & 23 & 17 & B2 & 7 \\
\hline $6 p 21.3$ & CYP21 & cytochrome P450 & 20 & $\mathrm{~L}^{\prime}$ & 23 & 17 & - & 7 \\
\hline $6 \mathrm{p} 21.3$ & C4 & complement component 4 & 20 & $\mathrm{~L}$ & 23 & 17 & - & 7 \\
\hline $6 p 21.3$ & TNFA & tumor necrosis factor, $\mathrm{A}$ & 20 & $S$ & 23 & 17 & - & $7 p 1.1-q 1.1$ \\
\hline $6 q 13$ & COL9A1 & collagen type IX, $\alpha-1$ & $20 q 24$ & S & - & 1 & - & - \\
\hline $6 q 24-p 23$ & EDN1 & endothelin 1 & 20 & $S$ & - & 13 & - & $7 \mathrm{p} 1.3-\mathrm{p} 1.2$ \\
\hline $6 q 12$ & ME1 & malic enzyme & $10 q 12$ & $S, I$ & 9 & 9 & B2 & 1 \\
\hline $6 q 21.1-q 23$ & CGA & glycoprotein hormone, $\alpha$ chain & 10 & 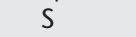 & 9 & 4 & - & 1 \\
\hline $6 q 21-q 22.3$ & COL10A1 & collagen, type $X, \alpha 1$ & 10 & S & - & 10 & - & - \\
\hline $6 q 25.1$ & ESR & estrogen receptor & $31 q 15$ & S & 9 & 6 & - & $1 \mathrm{p} 2.5-\mathrm{p} 2.4$ \\
\hline $6 q 26$ & PLG & plasminogen & 31 & S & - & 17 & - & - \\
\hline
\end{tabular}


Table 3. (Continued)

\begin{tabular}{|c|c|c|c|c|c|c|c|c|}
\hline $\mathrm{Hsa}^{\mathrm{a}}$ & Locus & Locus name & $E C A^{\mathbf{b}}$ & Method $^{c}$ & $B t a^{\mathrm{d}}$ & $M m u^{\mathrm{e}}$ & $F c a^{f}$ & $\mathrm{Ssc}^{\mathrm{g}}$ \\
\hline 7p15-p14 & $T C R G$ & T-cell receptor, $\gamma$ subunit & 4 & $S$ & 4 & 13 & $\mathrm{~A} 2$ & - \\
\hline $7 \mathrm{q} 11.2$ & ELN & elastin & 13 & $\mathrm{~S}$ & 25 & 5 & - & - \\
\hline $7 q 11.23-q 21$ & GUSB & glucuronidase, $\beta$ & 13 & $\mathrm{~S}$ & 25 & 5 & E3 & - \\
\hline $7 q 31.3$ & LEP & leptin & 4 & $\mathrm{~S}$ & 4 & 6 & - & 18 \\
\hline $7 q 36$ & EN2 & Engrailed 2 & 4 & $S$ & - & 5 & - & - \\
\hline $8 q 11$ & DNAPK & DNA protein kinase, catalytic sub. & $9 p 12$ & $S$ & - & 16 & - & - \\
\hline $8 q 24$ & & thyroglobulin & 9 & $S$ & 14 & 15 & - & - \\
\hline $9 p 22$ & IFNA1 & interferon, $\alpha-1$ & 23 & $S$ & 8 & 4 & - & 1 \\
\hline $9 \mathrm{p} 21$ & IFNB1 & interferon, $\beta-1$ & 23 & $\mathrm{~S}$ & 8 & 4 & - & 1 \\
\hline $9 \mathrm{p} 13$ & CNTFR & ciliary neurotrophic factor receptor & 23 & $\mathrm{~s}$ & $8 q 21$ & 4 & - & - \\
\hline $9 q 31$ & TXN & thioredoxin & 25 & $S$ & 8 & 4 & - & - \\
\hline $9 q 33$ & GRP78 & glucose-regulated protein & 25 & $S$ & 11 & 2 & - & $1 q 2.12 q 2.13$ \\
\hline $10 \mathrm{p} 13$ & VIM & vimentin & 29 & $S$ & 13 & 2 & - & 10 \\
\hline $11 \mathrm{p} 15.3-\mathrm{p} 15.1$ & PTH & parathyroid hormone & 7 & $S$ & 15 & 7 & - & 2 \\
\hline $11 \mathrm{p} 15.5$ & $H B B$ & hemoglobin $\beta$ & 7 & $\mathrm{~S}$ & 15 & 7 & D1 & - \\
\hline $11 \mathrm{p} 15$ & $T U B$ & Tubby & 7 & S & - & 7 & - & - \\
\hline $11 \mathrm{p} 15.5$ & IGF2 & insulin-like growth factor 2 & $12 q 14$ & S,I & 29 & 7 & - & - \\
\hline $11 \mathrm{q} 12$ & $C D 20$ & CD20 antigen & 12 & $S^{\prime \prime}$ & - & 19 & - & - \\
\hline 11 cen-q13 & $A D R B K 1$ & adrenergic receptor $\beta$ kinase 1 & 12 & $\mathrm{~s}$ & - & 19 & - & - \\
\hline $11 q 22$ & $P R$ & progesterone receptor & 7p16 & I & - & 9 & - & - \\
\hline $11 \mathrm{q} 23.3$ & THY1 & Thy- 1 T-cell surface antigen & 7 & S & 15 & 9 & - & - \\
\hline $11 q 23$ & DRD2 & dopamine receptor D2 & 7 & $S$ & - & 9 & & - \\
\hline $12 \mathrm{p} 12.2-\mathrm{p} 12.1$ & $\angle D H B$ & lactate dehydrogenase B & [28] & $S$ & 5 & 6 & B4 & $5 q 12$ \\
\hline $12 q 13.3$ & PFKM & phosphofructokinase m & [6] & $\mathrm{S}$ & 5 & - & - & - \\
\hline $12 \mathrm{q} 21$ & PEPB & peptidase B & [28] & $\mathrm{S}$ & 5 & 10 & B4 & 5 \\
\hline $12 q 22-q 24.1$ & IGF1 & insulin-like growth factor 1 & {$[28]$} & $S$ & 5 & 10 & - & $5 q 2.5$ \\
\hline $12 q 24$ & IFNG & interferon $\gamma$ & [6] & $S$ & 5 & 10 & - & $5 p 1.1-q 1.1$ \\
\hline $12 q 24.2$ & TCF1 & transcription factor 1 & [8] & $S$ & - & 5 & - & - \\
\hline $13 q 14.1-q 14.2$ & RB1 & retinoblastoma & 17 & $S$ & 12 & 14 & - & - \\
\hline $14 q 11$ & MYH6 & myosin, heavy 6 & 1 & $\mathrm{~s}$ & - & 14 & - & - \\
\hline $14 \mathrm{q} 11.2$ & $\mathrm{CHY}$ & Chymase, mast cell & 1 & $\mathrm{~s}$ & - & 11 & - & - \\
\hline $14 q 13.1$ & & nucleoside phosphorylase & $1 q 26$ & $S, I$ & 10 & 14 & B3 & $7 q 2.1-q 2.2$ \\
\hline $14 q 32.1$ & $P I / A A T$ & protease inhibitor/AAT & $24 q 15$ & $\mathrm{~L}, \mathrm{I}$ & 7 & 12 & - & $7 q 2.4-q 2.6$ \\
\hline 15q22-qter & MPI & mannose phosphate isomerase & 1 & $S$ & 21 & 9 & B3 & 7 \\
\hline $15 q 22-$ qter & CYP1A2 & cytochrome P450, subf. I, polyp. 2 & 1 & $S$ & - & 9 & - & - \\
\hline $15 q 25-q 26$ & IGF1R & insulin-1 growth factor 1 receptor & 1 & S & 21 & 7 & - & $1 \mathrm{q} 1.7-\mathrm{q} 1.2$ \\
\hline $15 q 26.1$ & $\mathrm{IDH}_{2}$ & isocitrate dehydrogenase 2 & 1 & S & - & 7 & B3 & - \\
\hline $15 q 26.1$ & FES & feline sarcoma virus homologue & 1 & $S$ & 21 & 7 & B3 & - \\
\hline 16 pter-p13.3 & $H B A$ & hemoglobin A & $13 q$ & $\mathrm{I}, \mathrm{L}$ & - & 11 & - & - \\
\hline $16 \mathrm{p} 24.3$ & MC1R & melanocortin receptor & $3 p 12$ & $\mathrm{~L}, \mathrm{~S}, \mathrm{I}$ & 18 & 8 & - & - \\
\hline $16 q 13-q 22.1$ & CES1 & carboxylesterase & 3 & $\mathrm{~L}$ & - & 8 & - & - \\
\hline $16 q 21$ & GOT2 & glutamate oxaloact. transam., mito & 3p15 & $\mathrm{I}, \mathrm{L}$ & - & 8 & - & - \\
\hline $16 q 22.1$ & $H P$ & haptoglobin & 3 & $\mathrm{~L}$ & 18 & 8 & - & - \\
\hline $17 q 11.2$ & EVI2A & ecotropic viral integration site $2 \mathrm{~A}$ & 11 & $\mathrm{~S}$ & - & 11 & - & - \\
\hline $17 q 11.2$ & NF1 & neurofibromatosis 1 & 11 & $\mathrm{~S}$ & 19 & 11 & - & - \\
\hline $17 q 21$ & $M Y L 4$ & myosin, light chain 4 & 11 & $S$ & 19 & 11 & & \\
\hline $17 q 22-q 24$ & $\mathrm{GH}$ & growth hormone & 11 & $S$ & 19 & 11 & - & $12 \mathrm{p} 1.2-\mathrm{p} 1.5$ \\
\hline $17 q 23.1-q 25.3$ & HYPP & hyperkalemic periodic paralysis & 11 & $\mathrm{~S}$ & - & 11 & - & - \\
\hline $17 q 25$ & P4HB & prolyl-4 hydroxylase $\beta$ & 11 & $\mathrm{~S}$ & 19 & 11 & - & - \\
\hline
\end{tabular}

(Continued on following page.) 
Table 3. (Continued)

\begin{tabular}{|c|c|c|c|c|c|c|c|c|}
\hline $\mathrm{Hsa}^{\mathrm{a}}$ & Locus & Locus name & $E C A^{\mathbf{b}}$ & Method $^{c}$ & $B t a^{\mathrm{d}}$ & $M m u^{\mathrm{e}}$ & $F c a^{f}$ & $\mathrm{Ssc}^{\mathrm{g}}$ \\
\hline $18 p 11.32$ & TS & thymidylate synthetase & [8] & $S$ & 24 & 5 & - & - \\
\hline $19 \mathrm{p} 13.3-\mathrm{p} 13.2$ & C3 & complement component 3 & 7pter & $\mathrm{I}, \mathrm{S}$ & - & 17 & - & $2 p 17-p 14$ \\
\hline 19cen-q13.2 & $A 1 B G$ & $\beta$ globulin, A1 & 10 & $\mathrm{~L}$ & - & - & - & - \\
\hline $19 q 13.1$ & GPI & glucose phosphate isomerase & 10pter & I & 18 & 7 & - & $6 q 12$ \\
\hline $19 q 13$ & $C R C$ & calcium release channel & 10pter & 1 & 18 & - & - & $6 q 12$ \\
\hline $19 q 13$ & CKM & creatine kinase, muscle & 10 & $S$ & - & 7 & - & - \\
\hline 20pterp12 & PRNP & prion protein & 22 & $S$ & 13 & 2 & - & - \\
\hline $20 q 11.2$ & GHRH & growth hormone releasing hormone & 22 & $S$ & 13 & 2 & - & - \\
\hline $20 q 11.2$ & $A S P$ & agouti signaling protein & $22 q 15$ & $S, I$ & - & 2 & - & - \\
\hline $20 q 13.11$ & $A D A$ & adenosine deaminase & 22 & S & 13 & 2 & A3 & - \\
\hline $20 q 13.2$ & GNAS1 & guanine nucleotide-binding protein & 22 & $S$ & 13 & 2 & - & - \\
\hline $21 q 22.3$ & MX1 & myxovirus resistance & $26 q 17$ & I & 1 & 16 & - & 13 \\
\hline $21 q 22.3$ & ETS2 & avian erythroblastosis virus onc. 2 & $26 q 17$ & $\mathrm{I}, \mathrm{S}$ & 1 & 16 & $\mathrm{C} 2$ & - \\
\hline 22q13.31-qter & ARSA & arylsulfatase $\mathrm{A}$ & [28] & $\mathrm{S}$ & - & 15 & - & - \\
\hline $22 q 13.1$ & CYP2D & cytochrome P450, subfamily IID & {$[28]$} & $S$ & 5 & 15 & - & - \\
\hline Xp11.21 & ALAS2 & anemia, hereditary sideroblastic & $\mathrm{x}$ & S & - & $\mathrm{x}$ & - & - \\
\hline $\mathrm{Xq} 27.1-\mathrm{q} 27.2$ & F9 & coagulation factor 9 & $\mathrm{x}$ & $S$ & $\mathrm{x}$ & $\mathrm{x}$ & $\mathrm{X}$ & $\mathrm{X}$ \\
\hline $\mathrm{Xq28}$ & G6PD & glucose 6 phosphate dehydrogenase & $\mathrm{X}$ & $\mathrm{S}$ & $\mathrm{X}$ & $\mathrm{x}$ & $\mathrm{X}$ & $\mathrm{X}$ \\
\hline $\mathrm{Xq} 28$ & $B G N$ & biglycan & $\mathrm{x}$ & S & - & $\mathrm{x}$ & - & - \\
\hline $\mathrm{Xq} 28$ & F18 & factor 18 & Xq29 & $S, I$ & - & - & - & - \\
\hline
\end{tabular}

a'Data retrieved from Online Mendelian Inheritance in Man (OMIM), Johns Hopkins University, Baltimore, MD (March 1999). Full

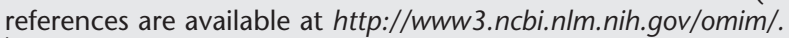

${ }^{\mathrm{b}}$ ECA assignment as in Shiue et al. (1999). In bold, physically assigned markers.

"Method: (I) In situ hybridization; (L) linkage; (S) synteny. Full references available at http://www.vgl.ucdavis.edu/horse.

${ }^{\mathrm{d} D a t a}$ retrieved from Bovmap Database, INRA, Laboratoire de Génétique Biochimique et de Cytogénétique de Jouy-en-Josas (March 1999). Full references are available at http://locus.jouy.inra.fr/cgi-bin/bovmap/intro.pl. and The Cattle Mapping Database (ARKDB), Roslin Institute, UK (March 1999). Full references available at http://www.ri.bbsrc.ac.uk/cgi-bin/arkdb/browsers/browser. sh?species=cattle

eData retrieved from Mouse Genome Informatics (MGI) Resource, Mouse Genome Informatics, The Jackson Laboratory, Bar Harbor, Maine (March 1999). Full references are available at http://www.informatics.jax.org/.

fData retrieved from O'brien et al. (1997b).

9Data retrieved from PiGBASE, Roslin Institute, Edinburgh, UK (May 1998). Full references are available at http://www.ri.bbsrc.ac. uk/pigmap/pigbase/pigbase.html.

\section{Provisional Assignment of Equine Synteny Groups by Comparative Mapping}

The lack of markers physically mapped to certain horse chromosomes has not allowed for the assignment of all 33 established equine synteny groups to their respective chromosomes. Nonetheless, the consideration of the synteny assignments of the equine type I loci we mapped and the other markers mapped with the UC Davis SCH panel, in conjunction with the data obtained by Zoo-FISH studies of the horse karyotype have allowed the tentative assignment of nine equine synteny groups.

A total of four equine type I loci were mapped to synteny group UCD5 (AT3, GBA, LAMC1, NGFB). The corresponding human homologs of these genes have been mapped to human Chromosome 1 (Table 1). Raudsepp et al. (1996) reported that painting probes from $H s a 1$, hybridize to three horse chromosomes (ECA2, 5, and 30). The previous assignment of UCD synteny groups 2 and 30 to ECA2 and ECA30, respectively (Shiue et al. 1999), therefore suggests that UCD5 is located on horse chromosome 5.

Four equine genes were assigned to UCD synteny group D (Table 2). The human homologs of equine FN1 and $C H R N G$ have been mapped to Hsa2, and the homologs of PFKM and IFNG have been mapped to $H s a 12$. Furthermore, we have recently assigned $P A X 3$ (Hsa2q35) and PMEL17 (Hsa12q13) to this synteny group and physically mapped PMEL17 to ECA6 by FISH (A.T. Bowling et al., unpubl. observations). The q arm of horse Chromosome 6 was shown to hybridize to painting probes from $H s a 12$, whereas the $\mathrm{p}$ arm did not hybridize to probes from any human chromosome (Raudsepp et al. 1996). Therefore, the assignment of UCD-D to horse Chromosome 6 is in agreement with ZooFISH studies of the horse karyotype and suggests that the $\mathrm{p}$ arm of ECA6 contains material orthologous to $H s a 2$. 
We had previously assigned UCD6 to ECA6 (Shiue et al. 1999) based on the assignment of a microsatellite (ASB14) by FISH (Breen et al. 1997), but this assignment has now been withdrawn (M. Breen, pers. comm.). Two horse genes, TCF1 (Hsa12) and TS (Hsa18), were assigned to UCD6 (Table 2). Therefore, the consideration that ECA8 is the only horse chromosome to hybridize to painting probes from human Chromosomes 12 and 18 (Raudsepp et al. 1996), and that no synteny groups have been assigned to this horse chromosome, suggests that UCD6 should be tentatively assigned to ECA8.

Painting probes from human Chromosomes 3, 9, 10 , and 13 were shown to hybridize exclusively to horse Chromosomes 16 and 19, 23 and 25, 1 and 29, and 17, respectively (Raudsepp et al. 1996). Equine genes with homologs located on these human chromosomes were assigned to UCD synteny groups 16 and 19, 23 and 25, 29, and 17, respectively (Table 2). The consideration that synteny groups UCD1, UCD19, and UCD23 were assigned respectively to ECA1, ECA19, and ECA23 (Shiue et al. 1999), suggests the provisional assignment of UCD synteny groups 16, 17, 25, and 29 to ECA16, ECA17, ECA25, and ECA29, respectively. The assignment of equine ADRB2 (Hsa5) to synteny group UCD14 is in agreement with the tentative assignment of this synteny group to horse Chromosome 14 as proposed by Caetano et al. (1999b).

Equine gene homologs mapping to human Chromosomes 12 and 22 were assigned to UCD synteny group C. Three horse chromosomes (ECA1, ECA8, and ECA28) have been shown to have blocks orthologous to Hsa12 and Hsa22 (Raudsepp et al. 1996). As mentioned previously, UCD1 has been assigned to ECA1 (Shiue et al. 1999) and we have tentatively assigned UCD6 to ECA8. Therefore, the most consistent explanation of the current data is the provisional assignment of UCDC to horse Chromosome 28.

In view of the described data, ECA27 is the only horse chromosome without an assigned or provisionally assigned synteny group. UCDA is the only established synteny group that has not been assigned to a horse chromosome. Therefore, the tentative assignment of UCDA to horse Chromosome 27 is reasonable, given the current data.

In addition, the assignments of type I loci to horse synteny groups have allowed two refinements of the human-horse comparative data produced with in situ hybridization experiments. The $\mathrm{q}$ arm of ECA13 was shown to be orthologous to Hsa16, whereas the orthology of the p arm of this horse chromosome was not determined (Raudsepp et al. 1996). The human homologs of two equine genes (ELN and GUSB) assigned to ECA13 have been mapped to $H s a 7$, which therefore suggests that the $\mathrm{p}$ arm of this horse chromosome contains material orthologous to human Chromosome 7.
Similarly, the orthology of the p arm of ECA6 could not be determined (Raudsepp et al. 1996) but the assignment of equine genes with homologs located on Hsa2 to ECA6 (UCDD), suggests that the p arm of this horse chromosome contains material orthologous to human Chromosome 2. Physical mapping of these equine genes mapped by synteny will be necessary to confirm these inferences.

\section{Application of Universal Primers for Type I Loci}

Although the published universal primers used for generating type I equine markers were highly useful for producing a first generation comparative map of the horse genome with the techniques used, additional markers could be readily developed from a few of the remaining primers tested. A total of 36 of the primers tested amplified candidate fragments for equine type I loci which could not be amplified in the somatic cell hybrids, while mouse-specific bands were amplified instead, and therefore could not be used for synteny mapping. We speculate that small deviations of the equine gene sequence from the consensus at the primer binding sites caused the observed preferential amplification of mouse products. Further characterization by cloning and sequencing of horse-specific PCR products from these primers, followed by redesigning of horse-specific primers, could result in additional markers for equine type I loci useful for synteny mapping.

It is likely that the observed amplification of no fragments, multiple fragments, and anonymous equine sequences by a number of the primers used in this study may have been caused by deviations of the equine gene sequences from the consensus sequences used for designing the primers. In addition, expansion of intron sequences within targeted gene regions to sizes beyond the amplification range of Taq DNA polymerase may also have caused the observed results. In these instances, markers for equine type I loci may be developed by redesigning new universal primers to target other intron regions of the respective genes using the reported strategies (Venta et al. 1996; Lyons et al. 1997).

\section{General Considerations}

Because synteny mapping by $\mathrm{SCH}$ panel analysis does not allow for the precise determination of the relative order and distance between syntenic markers, further work is needed to integrate the markers from this study into the International Equine Linkage Map (Guérin et al. 1999). Intraspecific sequence variation at the intron sequences amplified in most of the markers we mapped can be used to produce genetic markers useful for linkage mapping studies with pedigreed families (Lyons et al. 1997), and therefore order these genes relative to each other and to other markers, in the horse linkage 
map. In addition, dinucleotide repeat sequences found in the intron regions of a few of the equine PCR products we sequenced are currently being characterized (Caetano 1999) and may be useful in linkage studies as markers for the respective loci. The integration of type I loci into the horse linkage map will be useful for further refining the comparative map of the horse and revealing existing intrachromosomal rearrangements which may have occurred during the evolution of the horse genome.

The assignment of synteny groups to horse chromosomes has been highly dependent on the physical mapping of type I and II markers by FISH. Additional physical mapping data is needed to confirm proposed assignments, ratify provisional assignments, and moreover, to proceed with the establishment of a basic framework map of the horse genome. The small average size of the PCR-based type I markers we characterized precludes their direct use as probes in in situ hybridizations to metaphase chromosome spreads. The technical requirements of current FISH procedures demand larger DNA probes, which can be isolated from available horse BAC libraries (Godard et al. 1998) with the equine markers we characterized. In addition to their use in physical mapping, the isolated large-insert genomic clones may also be used for isolating microsatellite sequences associated with the respective equine genes, which could then be used in linkage studies.

Although type I gene homologs from all human chromosomes have been assigned to the horse genome map (Table 3), no equine type I loci have been mapped to horse Chromosomes 27 and 30. Zoo-FISH was used to show that ECA30 hybridizes to DNA fragments from human Chromosome 1 (Raudsepp et al. 1996) but the homeology of ECA27 could not be determined with this technique. A continued effort to develop and map markers for equine type I loci will be necessary to fill the gaps in the horse-human comparative map and to continue its refinement.

\section{METHODS}

\section{Optimization of PCR Amplification Parameters}

The methods utilized for optimizing PCR conditions to amplify horse-specific fragments with each primer set for use in synteny mapping with a horse-mouse SCH panel have been described previously (Caetano et al. 1999a). Optimal conditions for amplifying 68 equine type I loci are indicated in Table 1 . Horse and mouse PCR products of the same length were subjected to a panel of 15 restriction enzymes (not shown), in the buffer and temperature conditions recommended by the suppliers, prior to electrophoresis. Restriction enzymes selected to distinguish these PCR products from the two species are indicated in Table 1 .

\section{Sequencing}

Plugs containing DNA fragments amplified from a thorough- bred horse with each of the primer sets selected for mapping were taken from agarose gels with glass Pasteur pipettes and incubated at room temperature in $75 \mu \mathrm{l}$ of low TE buffer (10 mM Tris- $\mathrm{HCl}, 0.1 \mathrm{~mm}$ EDTA, $\mathrm{pH}$ 8.0) overnight. This solution was used as template for a PCR reaction under the conditions used to amplify the original product. An aliquot from this PCR reaction was quantified in an agarose gel and the remaining was used for cloning (TA Cloning Kit, Invitrogen). A minimum of 3 clones from each fragment was sequenced with $\mathrm{ABI}$ Prism sequencing kits and sequencing products were analyzed with an ABI 377 automated sequencer. Large PCR fragments that could not be readily cloned with the techniques used were isolated from agarose gels with the QIAEXII kit (Qiagen) and sequenced by direct sequencing. A few of the type I markers characterized were not completely sequenced because of the presence of extensive intron sequences which could not be used for sequence comparisons with other species. Each sequence obtained was subjected to BLAST searches of GenBank at the National Center for Biotechnology Information internet server (http://www.ncbi.nlm.nih.gov/). Sequences from 68 equine genes were submitted to GenBank.

\section{Somatic Cell Hybrid Panel Analysis}

The establishment of the UC Davis SCH panel has been described (Shiue et al. 1999). DNA from the same 108 horsemouse heterohybridoma cell lines used in previous studies (Shiue et al. 1999; Caetano et al. 1999a,b) were used in this study. DNA from each cell line was amplified with each of the 68 primer sets (Table 1) and scored for the presence or absence of horse-specific fragments after electrophoresis. Amplification products obtained from each hybrid cell line with 26 primer sets were digested with the respective restriction enzymes prior to electrophoresis (Table 1). Correlation coefficients were calculated between all of the markers in the UC Davis SCH panel database and each of the 68 loci studied. A correlation value $\geq 0.70$ (Table 2 ) was accepted as evidence for synteny between two markers (Chevalet and Corpet 1986).

\section{ACKNOWLEDGMENTS}

A.R.C. was supported by a fellowship from Conselho Nacional de Desenvolvimento Cientifico e Tecnológico (CNPq), Brasília, Brazil.

\section{REFERENCES}

Andersson, L., A. Archibald, M. Ashburner, S. Audun, W. Barendse, J. Bitgood, C. Bottema, T. Broad, S. Brown, D. Burt et al. 1996. Comparative genome organization of vertebrates. The First International Workshop on Comparative Genome Organization. Mamm. Genome 7: 717-734.

Archibald, A.L., C.S. Haley, J.F. Brown, S. Couperwhite, H.A. McQueen, D. Nicholson, W. Coppieters, A. Van de Weghe, A. Stratil, A.K. Winterö et al. 1995. The PIGMaP consortium linkage map of the pig (Sus scrofa) Mamm. Genome 6: 157-175.

Bailey, E., K.T. Graves, E.G. Cothran, R. Reid, T.L. Lear, and R.B. Ennis. 1995. Synteny-mapping horse microsatellite markers using a heterohybridoma panel. Anim. Genet. 26: 177-180.

Bishop, M.D., S.M. Kappes, J.W. Keele, R.T. Stone, S.L. Sunden, G.A. Hawkins, S.S. Toldo, R. Fries, M.D. Grosz, J. Yoo, and C.W. Beattie. 1994. A genetic linkage map for cattle. Genetics 136: 619-639.

Bowling, A.T., L.V. Millon, and S. Dileanis. 1997. Physical mapping of genetic markers to chromosome 30 using a trisomic horse and evidence for maternal origin of the extra chromosome. Chrom. Res. 5: 429-431.

Breen, M., G. Lindgren, M.M. Binns, J. Norman, Z. Irvin, K. Bell, K. 
Sandberg, and H. Ellegren. 1997. Genetical and physical assignments of equine microsatellites-First integration of anchored markers in horse genome mapping. Mamm. Genome 8: 267-273.

Caetano, A.R. 1999. Comparative mapping of the horse (Equss caballus) genome by synteny assignment of type-I genes with a horse-mouse somatic cell hybrid panel. Ph.D. Dissertation, University of California, Davis.

Caetano, A.R., D. Pomp, J.D. Murray, and A.T. Bowling. 1999a. Comparative mapping of 18 equine type I genes assigned by somatic cell hybrid analysis. Mamm. Genome 10: 271-276.

Caetano, A.R., L.A. Lyons, T.F. Laughlin, S.J. O'Brien, J.D. Murray, and A.T. Bowling. 1999b. Equine synteny mapping of comparative anchor tagged sequences (CATS) from human chromosome 5. Mamm. Genome (in press).

Chevalet, C. and F. Corpet. 1986. Statistical decision rules concerning synteny or independence between markers. Cytogenet. \& Cell Genet. 43: 132-139.

Chaudhary, R., T. Raudsepp, X.Y. Guan, H.G. Zhang, and B.P. Chowdhary. 1998. Zoo-FISH with microdissected arm specific paints for HSA2, 5, 6, 16, and 19 refines known homology with pig and horse chromosomes. Mamm. Genome 9: 44-49.

Cockett, N.E., S.P. Jackson, T.L. Shay, D. Nielsen, S.S. Moore, M.R. Steele, W. Barendse, R.D. Green, and M. Georges. 1994. Chromosomal localization of the callipyge gene in sheep (Ovis aries) using bovine DNA markers. Proc. Natl. Acad. Sci. 91: 3019-3023.

de Gortari, M.J., B.A. Freking, R.P. Cuthbertson, S.M. Kappes, J.W. Keele, R.T. Stone, K.A. Leymaster, K.G. Dodds, A.M. Crawford, and C.W. Beattie. 1998. A second-generation linkage map of the sheep genome. Mamm. Genome 9: 204-209.

Georges, M. and L. Andersson. 1996. Livestock genomics comes of age. Genome Res. 6: 907-921.

Godard, S., D. Vaiman, A. Oustry, M. Nocart, M. Bertaud, S. Guzylack, J.-C. Meriaux, and E.P. Cribiu. 1997. Characterization, genetic, and physical mapping analysis of 36 horse plasmid and cosmid-derived microsatellites. Mamm. Genome 8: 745-750.

Godard, S., L. Schibler, A. Oustry, E.P. Cribiu, and G. Guérin. 1998. Construction of a horse BAC library and cytogenetical assignment of 20 type I and type II makers. Mamm. Genome 9: 633-637.

Grobet, L., L.J. Martin, D. Poncelet, D. Pirottin, B. Brouwers, J. Riquet, A. Schoeberlein, S. Dunner, F. Ménissier, J. Massabanda et al. 1997. A deletion in the bovine myostatin gene causes the double-muscled phenotype in cattle. Nat. Genet. 17: 71-74.

Guérin, G., E. Bailey, D. Bernoco, I. Anderson, D.F. Antczak, K. Bell, M.M. Binns, A.T. Bowling, R. Brandon, G. Cholewinski et al. 1999. Report of the International Equine Gene Mapping Workshop: Male linkage map. Anim. Genet. (in press).

Hudson, T.J., L.D. Stein, S.S. Gerety, J. Ma, A.B. Castle, J. Silva, D.K. Slonim, R. Baptista, L. Kruglyak, S.H. Xu et al. 1995. An STS-based map of the human genome. Science 270: 1945-1954.

Lear, T.L., M.H. Adams, N.D. Sullivan, K.J. McDowell, and E. Bailey. 1998a. Assignment of the horse progesterone receptor (PGR) and estrogen receptor (ESR1) genes to horse chromosomes 7 and 31, respectively, by in situ hybridization. Cytogenet. \& Cell Genet. 82: $110-111$.

Lear, T.L., M. Breen, F.A. Ponce de Leon, L. Coogle, E.M. Ferguson, T.M. Chambers, and E. Bailey. 1998b. Cloning and chromosomal localization of MX1 and ETS2 to chromosome 26 of the horse (Equus caballus). Chrom. Res. 6: 333-335.

Lear, T.L., L.D. Coogle, and E. Bailey. 1998c. Assignment of the horse mitochondrial glutamate oxaloacetate transaminase 2 (GOT2) and v-kit Hardy-Zuckerman 4 feline sarcoma viral oncogene homolog (KIT) to horse chromosome 3 by in situ hybridization. Cytogenet. \& Cell Genet. 82: 112-113.

Lindgren, G., K. Sandberg, H. Persson, S. Marklund, M. Breen, B. Sandgren, J. Carlstén, and H. Ellegren. 1998. A primary male autosomal linkage map of the horse genome. Genome Res. 8: 951-966.

Lyons, L.A., T.F. Laughlin, N.G. Copeland, N.A. Jenkins, J.E. Womack, and S.J. O'Brien. 1997. Comparative anchor tagged sequences (CATS) for integrative mapping of mammalian genomes. Nat. Genet. 15: 47-56.

Naylor, S.L. 1997. Construction and use of somatic cell hybrids. In Genome mapping (ed. P.H. Dear), pp. 125-163. Oxford University Press, Oxford, UK.

O'Brien, S.J., J.E. Womack, L.A. Lyons, K.J. Moore, N.A. Jenkins, and N.G. Copeland. 1993. Anchored reference loci for comparative mapping in mammals. Nat. Genet. 3: 103-112.

O'Brien, S.J., S.J. Cevario, J.S. Martenson, M.A.Thompson, W.G. Nash, E. Chang, J.A. Graves, J.A. Spencer, K.W. Cho, H. Tsujimoto, and L.A. Lyons. 1997a. Comparative gene mapping in the domestic cat (Felis catus). J. Hered. 88: 408-414.

O'Brien, S.J., J. Wienberg, and L.A. Lyons. 1997b. Comparative genomics: Lessons from cats. Trends in Genet. 13: 393-399.

Raudsepp, T., L. Frönicke, H. Scherthan, I. Gustavsson, and B.P. Chowdhary. 1996. Zoo-FISH delineates conserved chromosomal segments in horse and man. Chrom. Res. 4: 218-225.

Raudsepp, T., K. Otte, B. Rozell, and B.P. Chowdhary. 1997. FISH mapping of the IGF2 gene in horse and donkey: Detection of homoeology with HSA11. Mamm. Gen. 8: 569-572.

Rettenberger, G., G. Adbo, and G. Stranzinger. 1996. Zoo-FISH analysis in the horse, Equus caballus, detects regions homologous to human chromosomes 3 and 14. J. Anim. Breeding \& Genet. 113: $145-148$.

Rhodes, M., R. Straw, S. Fernando, A. Evans, T. Lacey, A. Dearlove, J. Greystrong, J. Walker, P. Watson, P. Weston et al. 1998. A high-resolution microsatellite map of the mouse genome. Genome Res. 8: 531-542.

Sakagami M., T. Tozaki, S. Mashima, K. Hirota, and H. Mukoyama. 1995. Equine parentage testing by microsatellite locus at chromosome 1q2.1. Anim. Genet. 26: 123-124.

Shiue, Y.-L. 1999. Construction of a horse (Equus caballus) synteny and comparative map based on type I and type II markers. Ph.D. Dissertation, University of California, Davis.

Shiue, Y.-L., L.A. Bickel, A.R. Caetano, L.V. Millon, R.S. Clark, M.L. Eggleston, R. Michelmore, E. Bailey, G. Guérin, S. Godard et al. 1999. A synteny map of the horse genome comprised of 240 microsatellite and RAPD markers. Anim. Genet. 30: 1-9.

Stewart, E.A., K.B. McKusick, A. Aggarwal, E. Bajorek, S. Brady, A. Chu, N. Fang, D. Hadley, M. Harris, S. Hussain et al. 1997. An STS-based radiation hybrid map of the human genome. Genome Res. 7: 422-433.

Tozaki, T., M. Sakagami, S. Mashima, K. Hirota, and H. Mukoyama. 1995. ECA-3: Equine (CA) repeat polymorphism at chromosome 2p1.3-4. Anim. Genet. 26: 283.

Venta, P.J., J.A. Brouillette, V. Yuzbasiyan-Gurkan, and G.J. Brewer. 1996. Gene-specific universal mammalian sequence-tagged sites: Application to the canine genome. Biochem. Genet. 34: 321-341.

Wakefield, M.J. and J.A.M. Graves. 1996. Comparative maps of vertebrates. Mamm. Genome 7: 715-716.

Williams, H., C.M. Richards, B.A. Konfortov, J.R. Miller, and E.M. Tucker. 1993. Synteny mapping in the horse using horse-mouse heterohybridomas. Anim. Genet. 24: 257-260.

Received July 15, 1999; accepted in revised form September 14, 1999.
Genome Research www.genome.org 


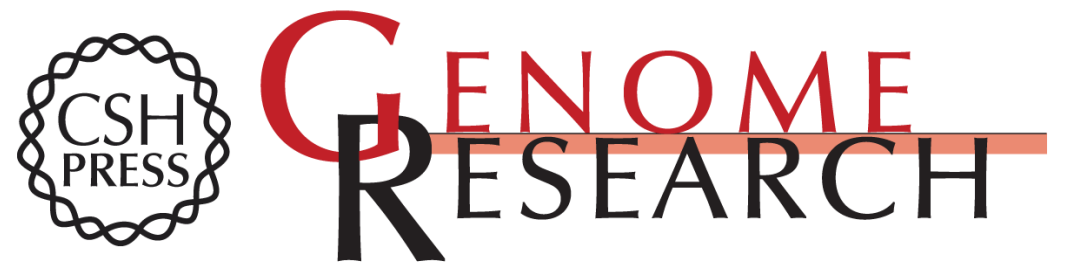

\section{A Comparative Gene Map of the Horse (Equus caballus)}

Alexandre R. Caetano, Yow-Ling Shiue, Leslie A. Lyons, et al.

Genome Res. 1999 9: 1239-1249

Access the most recent version at doi:10.1101/gr.9.12.1239

References This article cites 32 articles, 7 of which can be accessed free at:

http://genome.cshlp.org/content/9/12/1239.full.html\#ref-list-1

\section{License}

Email Alerting Receive free email alerts when new articles cite this article - sign up in the box at the Service top right corner of the article or click here.

\section{Affordable, Accurate Sequencing.}

To subscribe to Genome Research go to: https://genome.cshlp.org/subscriptions 